OPEN ACCESS

Edited by:

Mahmoud M. Alagawany,

Zagazig University, Egypt

Reviewed by:

Deanne Helena Hryciw,

Griffith University, Australia

Mahmoud Madkour,

National Research Centre, Egypt

${ }^{*}$ Correspondence:

Lin Yang

ylin898@scau.edu.cn

Yongwen Zhu

408034085@qq.com

Specialty section: This article was submitted to

Avian Physiology,

a section of the journal

Frontiers in Physiology

Received: 27 December 2021

Accepted: 31 January 2022

Published: 21 February 2022

Citation:

Zhang X, Wu Q, Zheng W, Liu C, Huang L, Zuo X, Xiao W, Han X,

Ye $H$, Wang W, Zhu Y and Yang L (2022) Exogenous Linoleic Acid Intervention Alters Hepatic Glucose Metabolism in an Avian

Embryo Model.

Front. Physiol. 13:844148.

doi: 10.3389/fphys.2022.844148

\section{Exogenous Linoleic Acid Intervention Alters Hepatic Glucose Metabolism in an Avian Embryo Model}

\author{
Xiufen Zhang ${ }^{1}$, Qilin Wu ${ }^{1}$, Wenxuan Zheng ${ }^{1}$, Chuang Liu ${ }^{2}$, Liang Huang ${ }^{1}$, Xin Zuo ${ }^{2}$, \\ Wenquan $\mathrm{XiaO}^{2}$, Xiaofeng $\mathrm{Han}^{2}$, Hui Ye ${ }^{1}$, Wence Wang ${ }^{1}$, Yongwen Zhu ${ }^{1 *}$ and Lin Yang ${ }^{1 *}$ \\ ${ }^{1}$ Guangdong Provincial Key Laboratory of Animal Nutrition and Regulation, College of Animal Science, South China \\ Agricultural University, Guangzhou, China, ${ }^{2}$ Wen's Food Group Co., Ltd., Yunfu, China
}

In the present study, developmental changes of gluconeogenesis and glycolysis in an avian model were measured, and then the intervention effects of in ovo feeding (IOF) linoleic acid (LA) on hepatic glucose metabolism were evaluated. In Experiment 1, thirty fertilized eggs were sampled on embryonic days (E) of 16, 19, 22, 25, 28, 31, and thirty newly-hatched ducklings at hatch (E34 and E35). In Experiment 2, a total of 120 fertilized eggs (60 eggs for each group) were injected into the yolk sac with PBS as the control group and LA as the IOF LA group on E25. Twelve eggs were selected for sample collection on E28 and E31. Serum contents of glucose, pyruvate, and lactate increased $(p<0.05)$ linearly or quadratically from E16 to hatch, as well as hepatic glycogen and pyruvate contents. Hepatic mRNA expression related to energy homeostasis, gluconeogenesis, and glycolysis increased $(p<0.05)$ in embryogenesis, and the plateau period was presented on E25-E31. IOF LA decreased $(p<0.05)$ serum contents of glucose, triacylglycerol, cholesterol, and hepatic oleic acid, unsaturated fatty acids on E28, as well as the gene expression relative to gluconeogenesis. IOF LA increased $(p<0.05)$ pyruvate content in serum and liver, and hepatic gene expression relative to glycolysis on E31. In summary, hepatic gluconeogenesis and glycolysis were enhanced to meet the increasing energy demands of embryonic development during E25 - hatch. Exogenous LA intervention on E25 could inhibit hepatic gluconeogenesis and enhance glycolysis during the later developmental period, disrupting glucose embryonic homeostasis and energy status.

Keywords: avian embryo model, glucose homeostasis, linoleic acid, in ovo feeding, energy status

\section{INTRODUCTION}

The avian embryo derives all its nutrient requirements from the nutrient deposits in the fertile egg during incubation. Avian embryos make use of $\mathrm{O}_{2}$ accession for fatty acid (FA) oxidation to get energy with the vascular system and pulmonary development (Tazawa et al., 1983; Moran, 2007). However, energy metabolism shifts from yolk lipids to the predominant carbohydrate substrate when the embryo reaches the oxygen consumption plateau (Donaldson et al., 1991; De Oliveira et al., 2008). Thus, gluconeogenesis and glycolysis are enhanced during the latehatch stage to maintain the energy homeostasis as well as normal physiological status for embryonic growth and development (Hu et al., 2017; Wan et al., 2018). For example, glycolysis 
reduces glucose to pyruvate and adenosine triphosphate (ATP), and pyruvate is converted to lactate that can be recycled back to glucose in the liver via the Cori cycle once the oxygen is available (De Oliveira et al., 2008; Bolaños et al., 2010). In addition, avian embryos are much easier to maintain and manipulate than most other vertebrate species. Therefore, it is speculated that an avian embryo during the last phase of incubation could be considered as an ideal model to understand vertebrate development via altering glucose homeostasis.

In ovo feeding (IOF) is an efficient technique to evaluate the effects of the exogenous nutrients on embryonic development using an avian model (Givisiez et al., 2020). IOF can deliver early nutrients and additives to embryos via yolk sac, albumen, air sac, and amnion et al. (Das et al., 2021). Previous studies have shown the effects of IOF carbohydrates, amino acids, minerals, and vitamins on embryonic development, and these exogenous nutrients can change blood histology, modify the regulation of transcription of different genes, and improve hatchability and perinatal growth in embryos and neonates (Tangara et al., 2010; Yair et al., 2015; Chen et al., 2021). In addition, IOF supplements like probiotics, prebiotics, and synbiotics protect against harmful gut microbes through competitive exclusion and maintain intestinal immune homeostasis (Shehata et al., 2021). There are limited reports on the effects of FA interventions. Linoleic acid (LA) has been proved to be involved in the regulation of glucose homeostasis via the alteration of $\mathrm{FA}$ and glucose metabolism in vivo and in vitro (Hamilton and Klett, 2021). Dietary LA could decrease blood glucose in obese rats (Matravadia et al., 2016; Holmäng et al., 2018). Glucose acts as an important fuel source for embryonic development (Minhas and Khan, 2016). Therefore, the developmental changes of serum glucose and hepatic glycogen concentrations in embryogenesis were measured to determine the critical period of glucose metabolism in the current study. We hypothesized that LA may decrease embryonic serum glucose and the adverse effects of IOF LA on the alteration of glucose metabolism to regulate embryonic development was evaluated using the avian embryo model.

\section{MATERIALS AND METHODS}

\section{Ethics Statement}

The protocol was reviewed and approved by the Animal Care and Use Committee of South China Agricultural University with the following reference number: No. 20110107-1.

\footnotetext{
Abbreviations: ADP, Adenosine diphosphate; AMP, Adenosine monophosphate; AMPK, Adenosine monophosphate-activated protein kinase; ATP, Adenosine triphosphate; CHO, Cholesterol; CoA, Coenzyme A; FA, Fatty acid; FAMEs, Fatty acid methyl esters; HKDC, Hexokinase; IOF, In ovo feeding; LA, Linoleic acid; NAD, Nicotinamide adenine dinucleotide; PBS, Phosphate-buffered saline; PC, Pyruvate carboxylase; PCK, Phosphoenolpyruvate carboxykinase; PFKFB2, 6-Phosphofructo-2-kinase; PFKL, Phosphofructokinase; PKM, Pyruvate kinase M1/2; PRKAA1, Protein kinase AMP-activated catalytic subunit alpha 1; RTqPCR, Real-time quantitative PCR; SFAs, Saturated fatty acids; TG, Triacylglycerol; UFAs, Unsaturated fatty acids.
}

\section{Incubation and in ovo Feeding}

Fertilized Muscovy duck eggs were obtained from a commercial breeder farm (Wen's Food Group Co., Ltd., Yunfu, Guangdong, China), and all the eggs were collected from hens at 33 week of age belonging to the same breeder flock. The eggs were incubated in the automatic-controlled incubator (Dezhou Keyu Hatching Equipment Co., Ltd., Dezhou, China) according to standard hatchery practices $\left(37.5 \pm 0.5^{\circ} \mathrm{C}\right)$, relative humidity $(55 \pm 5 \%)$ until embryonic day 31 (E31). Next, all eggs were transferred to hatching crates and moved to hatchers which were set at a temperature of $37.0 \pm 0.5^{\circ} \mathrm{C}$ and declined to $36.0 \pm 0.5^{\circ} \mathrm{C}$ at the end of incubation.

In Experiment 1, a total of 350 Muscovy duck eggs were collected for incubation, and unfertilized and unviable eggs were discarded after candling on E15. A total of 180 viable eggs $(75.2 \pm 1.8 \mathrm{~g})$ and 30 hatchlings $(43.19 \pm 0.7 \mathrm{~g})$ were applied for the test (five embryos per replicate, $n=6$ ). Thirty viable eggs were selected for samples collection on E16, $\mathrm{E} 19, \mathrm{E} 22, \mathrm{E} 25, \mathrm{E} 28$, and E31, and 30 ducklings at hatch (E34 and E35). In Experiment 2, a total of 200 eggs were collected for incubation, and 120 viable eggs $(79.6 \pm 1.9 \mathrm{~g})$ were divided into two groups on E25, the control group and the treatment group, with 60 eggs each. The eggs in the control group or the treatment group were injected into the yolk sac with a volume of $100 \mu \mathrm{l}$ of phosphate-buffered saline (PBS, G4202, Servicebio, Wuhan, China) or LA ( $\geq 98 \%$, 62230, Sigma-Aldrich, Wyoming, United States), respectively. The IOF procedure was conducted as previously reported (Chen et al., 2020). In brief, the site for injection was found by illumination (about one-third from the sharp end of the egg) and disinfected with $75 \%$ ethanol before injection. A small hole $(0.8 \mathrm{~mm}$ in diameter) was drilled on the eggshell by a hand-hold pearl driller. The sterile disposable $25.0 \times 0.6 \mathrm{~mm}$ needle was attached to a $1.0 \mathrm{ml}$ syringe, which was replaced after each egg injection. The hole was sealed with medical adhesive tape $\left(1.0 \times 1.0 \mathrm{~cm}^{2}\right)$ immediately after injection, and the egg was transferred to the incubator. All eggs were kept outside the incubator for less than $30 \mathrm{~min}$ during the injection process. Twelve eggs were selected to collect samples (two embryos per replicate, $n=6$ ) on E28 and E31.

\section{Sample Collection}

Samples of serum and liver were pooled together for each replicate in Experiment 1 (five embryos) and Experiment 2 (two embryos). Blood samples were collected from the umbilical vein of the embryos using glass Pasteur pipettes $(7 \times 150 \mathrm{~mm})$. The tip of a glass Pasteur pipette was melted on the outer flame of an alcohol lamp and then withdrawn with a forcep to make a needle $<0.3 \mathrm{~mm}$ in diameter. Blood samples were collected from the jugular vein of ducklings using disposable syringes at hatch. The serum was separated by centrifugation (1-14, Sigma, Germany) at $664 \times g$ for $10 \mathrm{~min}$ at room temperature and then stored at $-20^{\circ} \mathrm{C}$ for biochemical index analysis. The liver samples were cleaned of extraneous tissues, rinsed with ice-cold PBS, and then frozen in liquid nitrogen and stored 
at $-80^{\circ} \mathrm{C}$ for analysis of biochemical index and relative expression of gene mRNA and protein.

\section{Biochemical Analysis}

The serum glucose was measured using an automatic biochemical analyzer (Roche Cobas C702, Basel, Switzerland). The contents of glycogen, pyruvate, lactate, triacylglycerol (TG), and cholesterol $(\mathrm{CHO})$ in serum or liver were measured according to the manufacturer's instructions for each assay kit (Nanjing Jiancheng Bioengineering Institute, Nanjing, China). The serum contents of adenosine phosphate and coenzyme were measured using the ELISA assay kits as follows (Shanghai Enzyme-linked Biotechnology Co., Ltd., Shanghai, China): ATP, adenosine diphosphate (ADP), adenosine monophosphate (AMP), acetyl coenzyme A (acetyl CoA), and malonyl coenzyme A (malonyl CoA). Hepatic nicotinamide adenine dinucleotide (NAD) contents were measured using the assay kit (Beyotime Biotechnology, Shanghai, China) including $\mathrm{NADH}$ (reduced form) and $\mathrm{NAD}^{+}$(oxidized form).

\section{Fatty Acids Analysis}

FA profiles were determined by using gas chromatography (7890A, Agilent Technologies, Santa Clara, CA, United States). Lipids from liver tissues (Experiment 2) were extracted with chloroform and methanol (2:1, vol/vol), and the separated lipid fraction was converted to FA methyl esters (FAMEs) by saponification using 0.5 M KOH-methanol, followed by methylation with $200 \mu \mathrm{l}$ boron trifluoride diethyl etherate (B104430, Sigma-Aldrich). Next, the FAMEs were mixed with $\mathrm{N}$-hexane and saturated sodium chloride solution, and the mixture was shaken for $10 \mathrm{~min}$ and centrifuged at $1,200 \times g$ for $10 \mathrm{~min}$. The upper phase was collected and dried with sodium sulfate for gas chromatographic analysis. The FAMEs were identified by comparing retention times to FAME standards (CRM47885, Sigma-Aldrich). The results of FA composition were reported as the percentage of total FA.

\section{Gene mRNA Relative Expression Analysis}

The total RNA isolation and the real-time quantitative procedure were conducted as previously reported (Kang et al., 2017; Madkour et al., 2021). Total RNA was extracted from the liver samples according to reagent protocols using a Trizol reagent (Invitrogen, Carlsbad, USA) followed by purification of total RNA using the kit with DNase treatment (Magen, Guangzhou, China). The RNA quality and quantity were determined using agarose gel electrophoresis (4.5\%) and NanoDrop 2000 (Thermo Scientific, Wilmington, DE, USA). One $\mu \mathrm{g}$ of total RNA was converted into cDNA by reverse transcription as described in Primer Script RT Reagent Kits (TaKaRa, Dalian, China). Primer sequences were obtained from GenBank (Table 1) and designed and synthesized by Sangon Biotechnology Co., LTD (Shanghai, China). The PCR products were analyzed by using agarose gel electrophoresis (4.5\%). Each qPCR reaction had a final volume of $10 \mu \mathrm{l}$ of the reaction mixture, which consisted of $5 \mu \mathrm{l}$ SYBR Green Realtime PCR Master Mix kit (QPK-201, TOYOBO, Osaka, Japan) with $3.2 \mu \mathrm{l}$ DNase/RNase-Free water, $0.4 \mu \mathrm{l}$ forward and reverse specific primers for each gene and $1 \mu \mathrm{l}$ of cDNA template. Real-time quantitative PCR was performed using the detection system (Applied Biosystems QuantiStudio 7 Flex, Life Technologies, Carlsbad, CA, United States). The following cycling conditions were used: $95^{\circ} \mathrm{C}$ for $1 \mathrm{~min}$, followed by 40 cycles each at $95^{\circ} \mathrm{C}$ for $15 \mathrm{~s}, 60^{\circ} \mathrm{C}$ for $15 \mathrm{~s}$, and $72^{\circ} \mathrm{C}$ for $45 \mathrm{~s}$. Melt-curve analysis was performed to verify the specificity of qPCR-amplified products. The quantification of the mRNA expression was calculated by the comparative CT method ( $2^{-\Delta \Delta C T}$; Livak and Schmittgen, 2001).

\section{Western Blotting Analysis}

The liver samples collected on E16, E22, E28, and hatch were used for western blotting analysis. The tissues were lysed with ice-cold RIPA lysis buffer containing $1 \mathrm{mmol} / \mathrm{l}$ phenylmethylsulfonyl fluoride. The lysates were placed on ice for $10 \mathrm{~min}$ and the homogenate was centrifuged at $12,000 \times g$ for $5 \mathrm{~min}$ at $4^{\circ} \mathrm{C}$. The protein concentration in the collected supernatant was measured using the bicinchoninic acid (BCA) assay kit (Beyotime Biotechnology, Shanghai, China). The protein supernatant was mixed with loading buffer, denatured for $5 \mathrm{~min}$ by heat shock at $95^{\circ} \mathrm{C}$, and then stored at $4^{\circ} \mathrm{C}$ for western blotting analysis. Proteins were separated via an electrophoretic SDS-PAGE on $10 \%$ gel. Next, proteins were transferred to a nitrocellulose membrane with Trans-Blot, and the membrane was blocked with a buffer containing $5 \%$ skim milk. Membranes were incubated overnight at $4^{\circ} \mathrm{C}$ with rabbit monoclonal antibodies for adenosine monophosphate-activated protein kinase alpha 1 (AMPK $\alpha 1$, ab32047, Abcam, Cambridge, England), glucose-6phosphatase (G6PC1, ab243319, Abcam), and $\beta$-actin (66009-1-Ig, Proteintech, Chicago, United States). Then, membranes were incubated with HRP goat anti-mouse IgG (SA00001-1, Proteintech) or HRP goat anti-rabbit IgG (SA00001-2, Proteintech) at room temperature. Proteins of interest were diluted in buffer with enhanced chemiluminescence solution. The bands were detected using a chemiluminescence imaging system (ChemiScope6100, Qinxiang, Shanghai China). The density of bands was determined using ImageJ software and the results were expressed as the intensity signal in arbitrary units after normalization.

\section{Statistical Analysis}

Data from Experiment 1 were statistically analyzed using the PROC GLM procedures for one-way ANOVA by Statistical Analysis System v9.2 (SAS Inst. Inc., Cary, NC, United States). When significant differences were found $(p<0.05)$, Duncan's multiple comparisons test was performed and orthogonal polynomial contrasts were used to identify the form of the effect (linear or quadratic) over time (incubation days). Data from Experiment 2 on each sampling time were analyzed with a $t$-test using the PROC Ttest procedure in SAS v9.2, and differences were considered to be significant at $p<0.05$.

\section{RESULTS}

\section{Biochemical Index Related to Glucose Metabolism (Experiment 1)}

The serum contents of glucose, pyruvate, and lactate increased $(p<0.05)$ in a linear and quadratic manner during embryogenesis 
TABLE 1 | Forward and reverse primer sequences for PCR analysis.

\begin{tabular}{|c|c|c|c|c|}
\hline Target genes & Forward primer $\left(5^{\prime} \rightarrow 3^{\prime}\right)$ & Reverse primer $\left(5^{\prime} \rightarrow 3^{\prime}\right)$ & Genebank accession no. & Product size (bp) \\
\hline PRKAA1 & GCGGCGGCGGATAAACAGAAG & CATGCTTGCCAACCTTGACTITGC & XM_027447031.2 & 112 \\
\hline$P C$ & CAACTACCTGCCCAACСTGCTG & GGCTGTATITGGTGCGTGTAGGG & NM_205471.1 & 119 \\
\hline PCK & GAGCCATTGCCACCAGGAGTAAC & GCAGAACCGTGAGTTGGGATGAG & XM_021276483.2 & 99 \\
\hline PFKFB2 & ACTTCTTCAGGCACGATAACAAGGAG & CGAGTTGTGTTGGTCGCATCAAAC & XM_038168182.1 & 136 \\
\hline G6PC1 & CTGGCTCAACCTCGTCTTCAAGTG & GGCGTTGCTGTAGTAGTTGGTCTC & XM_027445511.2 & 85 \\
\hline HKDC1 & AGGCAAGCCAGCATTGACAAGG & TCССTCAGCATATCAACAACATCTTCC & XM_013092011.4 & 98 \\
\hline PFKL & GTGGGTGCCGTGAGAGAAGTTG & TGTAGAGGAACTCGGTGGTGTAGTG & XM_038183738.1 & 84 \\
\hline$P K M$ & AGCCAACCATTGCGAGGAACAC & GTGGGTGCCGTGAGAGAAGTTG & XM_038184779.1 & 131 \\
\hline FASN & TCTCTGCCATCTCCCGAACTTCC & TTAGCCACTGTGCCAACTCAAGC & XM_027471234.2 & 96 \\
\hline SCD1 & AGTTCTCCTCCGCTTCCAGC & TTCTCCATGACGGCATCCCC & XM_027460089.2 & 82 \\
\hline PPAR $\alpha$ & ACCATCCTGATGATACCTTСCTCTTCC & AAGTTGAGCATGTTCTGTGACAAGTTG & NM_001310383.1 & 86 \\
\hline$R \times R \alpha$ & TGCGAGCCATTGTCCTCTTCAAC & GATGCGTACACCTTCTCCCGTAAC & XM_027471073.2 & 88 \\
\hline$\beta$-actin & TACGCCAACACGGTGCTG & GATTCATCATACTCCTGCTTG & NM_00131042.1 & 215 \\
\hline
\end{tabular}

PRKAA1, protein kinase AMP-activated catalytic subunit alpha 1; PC, pyruvate carboxylase; PCK, phosphoenolpyruvate carboxykinase; PFKFB2, 6-phosphofructo-2-kinase; G6PC1, glucose-6-phosphatase; HKDC, hexokinase; PFKL, phosphofructokinase; PKM, pyruvate kinase M1/2; FASN, fatty acid synthase; SCD1, stearoyl-CoA desaturase; PPARo, peroxisome proliferator-activated receptor alpha; $R X R \alpha$, retinoid $X$ receptor alpha.

(Figure 1A), reaching plateau periods during E28 - hatch, E31 - hatch, and E25-E31, respectively. The serum contents of ADP, acetyl CoA, and malonyl CoA increased $(p<0.05)$ linearly or quadratically (Figure 1A). Hepatic glycogen and pyruvate increased $(p<0.05)$ in a linear and quadratic manner, with the peak period of E28 - hatch or E28-E31, respectively (Figure 1B). As incubation day increased, hepatic NADH content decreased $(p<0.05)$ linearly and the ratio of $\mathrm{NAD}^{+}$to NADH increased $(p<0.05)$ linearly, with maximal changes observed at the period of E28 - hatch (Figure 1B).

\section{Hepatic Gene and Protein Expression Related to Glucose Metabolism (Experiment 1)}

Hepatic mRNA expression related to energy regulation, gluconeogenesis, and glycolysis of protein kinase AMP-activated catalytic subunit alpha 1 (PRKAA1), pyruvate carboxylase $(P C)$, phosphoenolpyruvate carboxykinase $(P C K), 6$-phosphofructo2-kinase (PFKFB2), G6PC1, hexokinase (HKDC), phosphofructokinase $(P F K L)$, and pyruvate kinase M1/2 (PKM) increased $(p<0.05)$ linearly or quadratically in response to the increased incubation day, and the plateau period was shown between E25 and E31 (Figure 2A). Hepatic protein expression of AMPK $\alpha 1$ and G6PC1 increased $(p<0.05)$ linearly or quadratically, and the higher level was presented between E22 and E28 (Figures 2B,C).

\section{Effect of IOF LA on Biochemical Index and Fatty Acids (Experiment 2)}

Compared to the control group, IOF LA group decreased $(p<0.05)$ serum contents of glucose, TG, and CHO on E28 and E31, and serum pyruvate content on E28 (Figure 3). IOF LA group increased $(p<0.05)$ serum contents of pyruvate, lactate, and acetyl CoA on E31, and hepatic pyruvate content on E28 and E31 (Figures 3A,B). For FA profiles, IOF LA group increased $(p<0.05)$ hepatic stearic acid $(\mathrm{C} 18: 0)$ and saturated FAs (SFAs) on E28, and myristic acid (C14:0) and
SFAs on E31, while IOF LA group decreased $(p<0.05)$ hepatic oleic acid (C18:1n-9), unsaturated FAs (UFAs), and the ratio of UFAs to SFAs on E28 by comparison to the control group (Table 2).

\section{Effect of IOF LA on Hepatic Gene Relative Expression (Experiment 2)}

IOF LA group decreased $(p<0.05)$ hepatic mRNA expression of PRKAA1, PC, PCK, PFKFB2, PFKL, FASN, SCD1, PPAR $\alpha$, and $R X R \alpha$ on E28, and increased $(p<0.05)$ mRNA expression of PRKAA1, HKDC, and PFKL on E31 (Figure 4).

\section{DISCUSSION}

Hepatic glucose metabolism is essential for energy homeostasis by balancing glucose storage and utilization during incubation. The blood glucose supply and muscle and hepatic glycogen storage were mainly mediated by the metabolic pathway of gluconeogenesis in embryogenesis (De Oliveira et al., 2008; Guo et al., 2013). In addition, both blood glucose and hepatic glycogen levels are considered as the criterion in assessing embryonic energetic status based on the data examined in carbohydrates metabolism (Christensen et al., 1999, 2000). Previous studies demonstrated that blood glucose concentration is positively associated with embryonic survival and hatchling weight (Christensen et al., 2000). Therefore, the avian embryo in the final stage of incubation has been considered as a suitable model for assessing the connection between glucose metabolism and energy status. In the present study, serum glucose and hepatic glycogen contents increased as incubation proceeded from E16 to hatch, and the plateau period was shown on the last week of the incubation (E28 hatch). These parallel changes of serum glucose and hepatic glycogen were also confirmed in chick embryos (Latour et al., 1996; Christensen et al., 2001; Roy et al., 2013a). The rate-limiting enzymes of PC, PCK, PFKFB2, and G6PC1 are responsible for the irreversible catalytic reactions in 
A

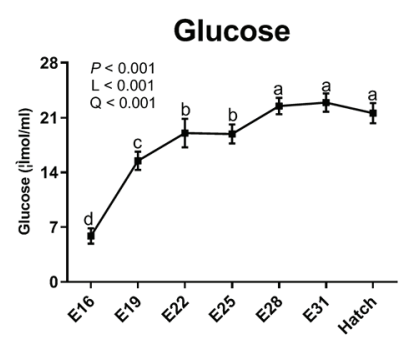

Acetyl CoA

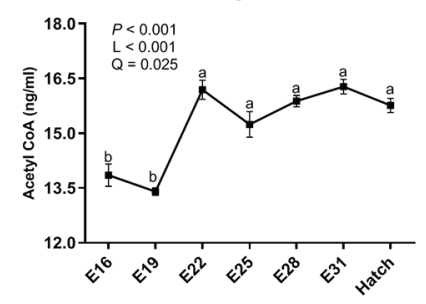

B

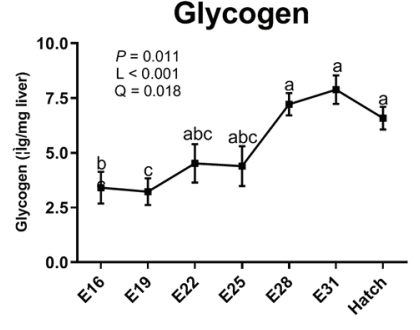

NAD ${ }^{+}$

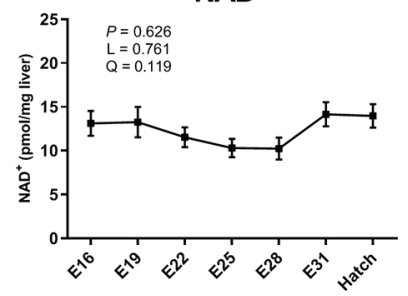

Pyruvate

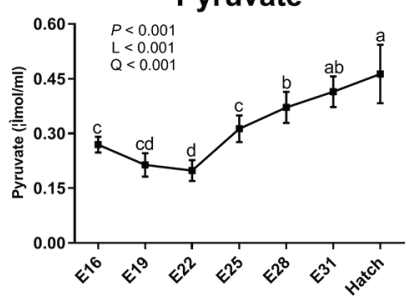

Malonyl CoA

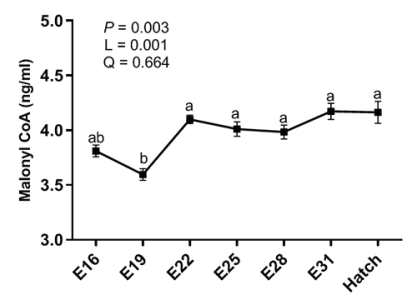

Pyruvate

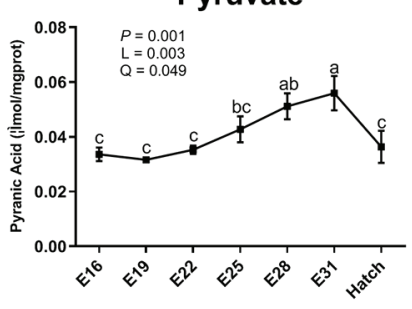

NADH

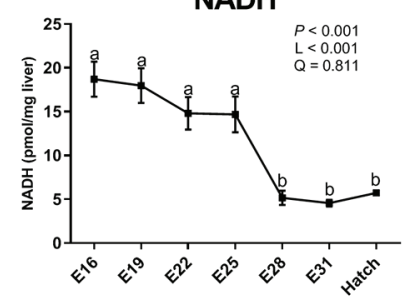

Lactate

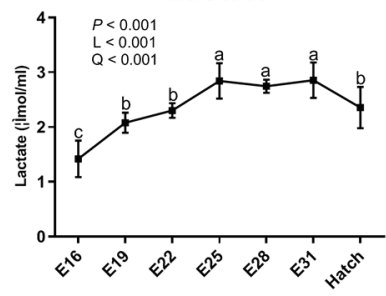

Adenosine Phosphate

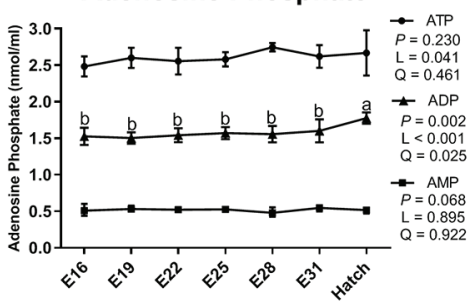

Lactate

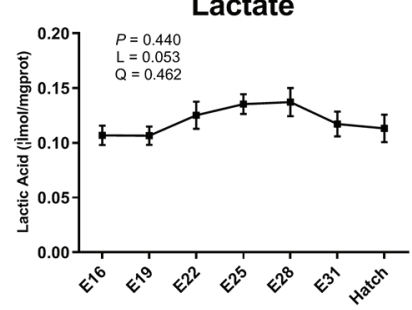

$\mathrm{NAD}^{+} / \mathrm{NADH}$

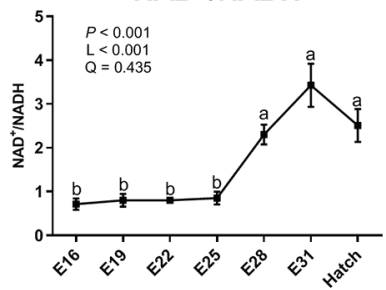

FIGURE 1 | Dynamic changes of serum and hepatic biochemical index in the avian model. (A) serum biochemical index; (B) hepatic biochemical index. Abbreviations: CoA, coenzyme A; ATP, adenosine triphosphate; ADP, adenosine diphosphate; AMP, adenosine monophosphate; NAD, nicotinamide adenine dinucleotide; $\mathrm{NAD}^{+}$, the oxidated form of NAD; NADH, the reduced form of NAD. Data are expressed as mean \pm SEM $(n=6)$, and values on the same line with different lowercase letters indicated statistically significant differences (one-way ANOVA, $P<0.05$ ). L, linear; $Q$, quadratic.

gluconeogenesis (Shen and Mistry, 1979; van Schaftingen and Gerin, 2002; Chesney, 2006). Hepatic gene mRNA expression of PRKAA1, PC, PCK, PFKFB2, and G6PC1, and the relative protein expression of $\mathrm{G} 6 \mathrm{PC} 1$ increased from E16 to hatch, and the maximal values were observed between E22 and E31. These results could partly explain the increasing serum glucose concentration and hepatic glycogen content during embryonic development. The avian embryo begins anaerobic catabolism of glucose before hatch, and glucose is metabolized into the highly versatile metabolite pyruvate (De Oliveira et al., 2008). And then, pyruvate can be anaerobically oxidized to lactate, accompanied by the conversion of NADH to $\mathrm{NAD}^{+}$(Bar-Even et al., 2012; Luengo et al., 2021). In the current study, glycolysis was enhanced as indicated by an increase in pyruvate and lactate contents in serum or liver and a decrease in hepatic NADH content during the last period of incubation (E28 - hatch). Glycolysis supplies the ATP molecules produced by a reversible reaction (2ADP $\leftrightarrow$ ATP + AMP; Noor et al., 2010; Schormann et al., 2019). The increase in ADP content implied that ATP was hydrolyzed to generate energy for the maintenance of embryonic growth and development. It's suggested that glycolysis provides an additional energy source (except for FA oxidation) for the greater demands during the later period of incubation, especially during internal piping (break the air cell by the beak) and external piping (break the eggshell) periods (Moran, 2007; De Oliveira et al., 2008). Glycolysis is regulated by the key enzymes of HKDC, PFKL, 


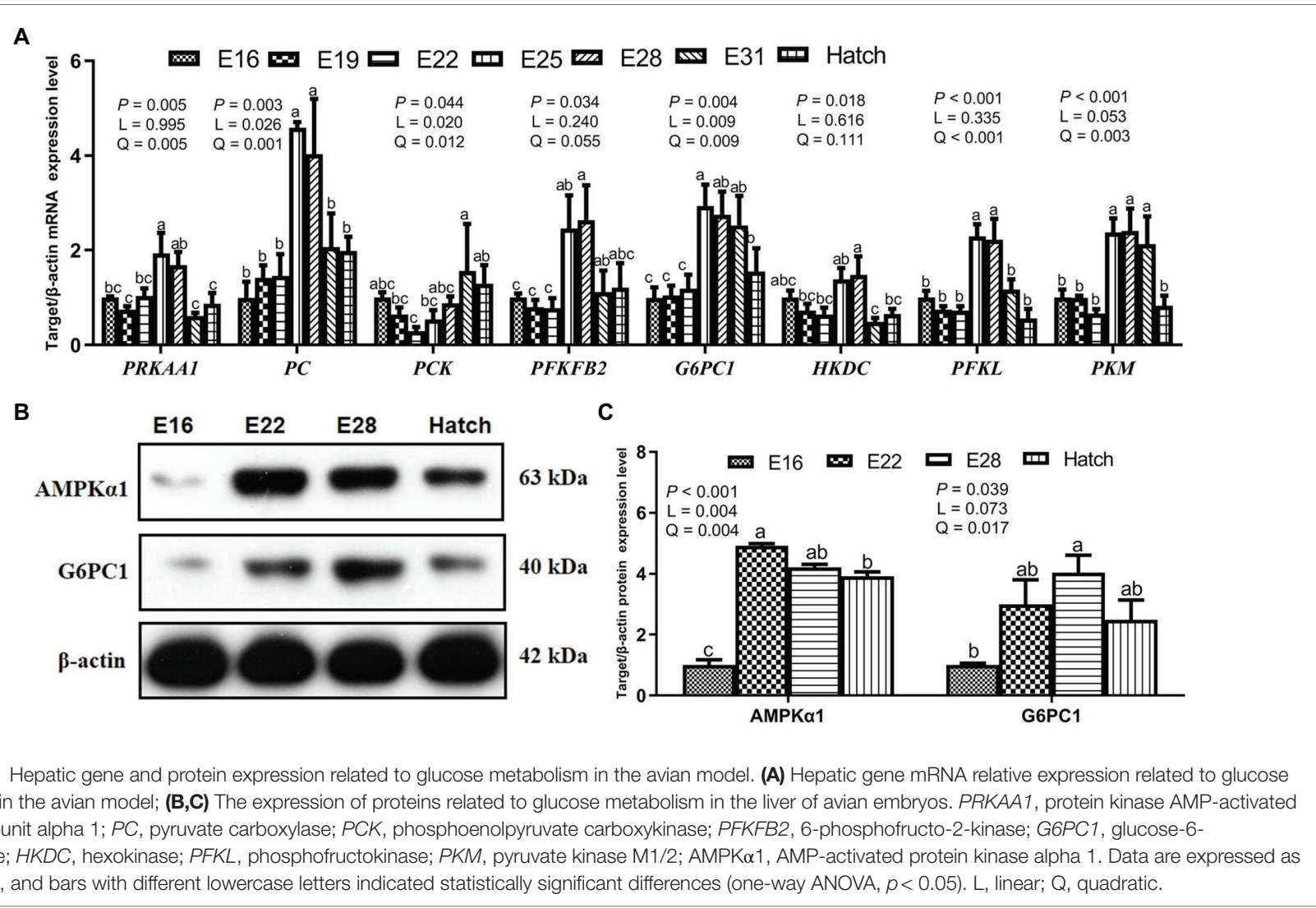

and PKM (Bolaños et al., 2010). Hepatic gene mRNA expression of $H K D C, P F K L$, and $P K M$ increased during embryonic development, and the plateau period was achieved between E25 and E31. A similar pattern was observed in chick and pigeon embryos reported previously (Roy et al., 2013b; Peng et al., 2018; Wan et al., 2018). Then, the increased protein expression level of AMPK $\alpha 1$ was observed in liver during E22 - E28. It's implied that the AMPK pathway is activated by falling energy status and promotes ATP production via increasing catabolism as well as switching off biosynthetic pathways (Hardie et al., 2012; Hardie, 2015). Briefly, these results suggested that the glucose homeostasis turnover between gluconeogenesis and glycolysis enhanced energy generation to meet the high energy requirements during embryonic development and as fuel storage for survival at the early post-hatch period (Li et al., 2008; Payne et al., 2019).

Previous studies have demonstrated that LA reduces glucose concentration in the diverse cell, animal, and human models (Conde-Aguilera et al., 2012; Matravadia et al., 2016; Andersson-Hall et al., 2018; Holmäng et al., 2018). In the present study, the intervention of exogenous LA led to a decrease in serum contents of TG and $\mathrm{CHO}$, and then reduced the concentration of glycerol, which was derived from TG hydrolysis and the main precursor of gluconeogenesis in late-term avian embryos (Sunny and Bequette, 2011; Neves et al., 2017; Xue et al., 2017). Moreover, IOF LA inhibited gluconeogenesis on E28 as displayed by a decrease in serum glucose concentration and gene expression of $P C, P C K$, and PFKFB2. However, IOF LA increased stearic acid (C18:0) and decreased oleic acid $(\mathrm{C} 18: 1 \mathrm{n}-9)$ in liver, due to the weakened activity of desaturases responsible for the synthesis of UFAs, especially the $\Delta 9$-desaturase activity (Kouba and Mourot, 1998; Bee, 2000; Novak et al., 2012; Lounis and Bergeron, 2017; Piccinin et al., 2019). Hepatic mRNA expression of FASN, SCD1, PPAR $\alpha$, and $R X R \alpha$ related to FA synthesis and oxidation was downregulated on E28. It's implied that FA oxidation could not satisfy all the energy demands for the embryos as the internal oxygen concentration became limited. In addition, IOF LA enhanced glycolysis on E31 as indicated by an increase in serum pyruvate and lactate contents, as well as the upregulation of hepatic gene expression of HKDC and PFKL. The synthesis of serum glucose and hepatic glycogen was suppressed, combined with the enhanced glycolysis, which led to a reduced energy fuel storage for late embryonic development. Hence, the embryonic mortality in IOF LA group (24.27\%) was increased by $8 \%$ compared to the control group (16.67\%). Similarly, in vivo studies showed that elevated maternal dietary LA (or conjugated LA) reduced fetal survival and increased embryonic mortality in rat or chicken models (Leone et al., 2010; Shrestha et al., 2019). It was concluded exogenous LA could inhibit hepatic gluconeogenesis and enhanced glycolysis, thus leading to the impairment of glucose homeostasis and energy status during embryonic development. 
A
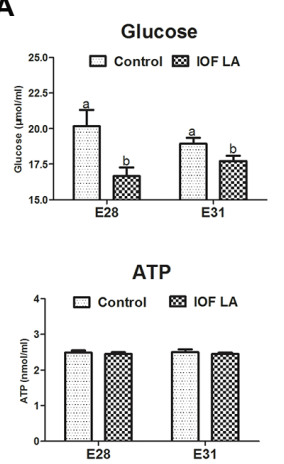

B
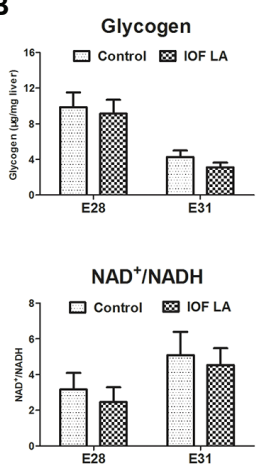
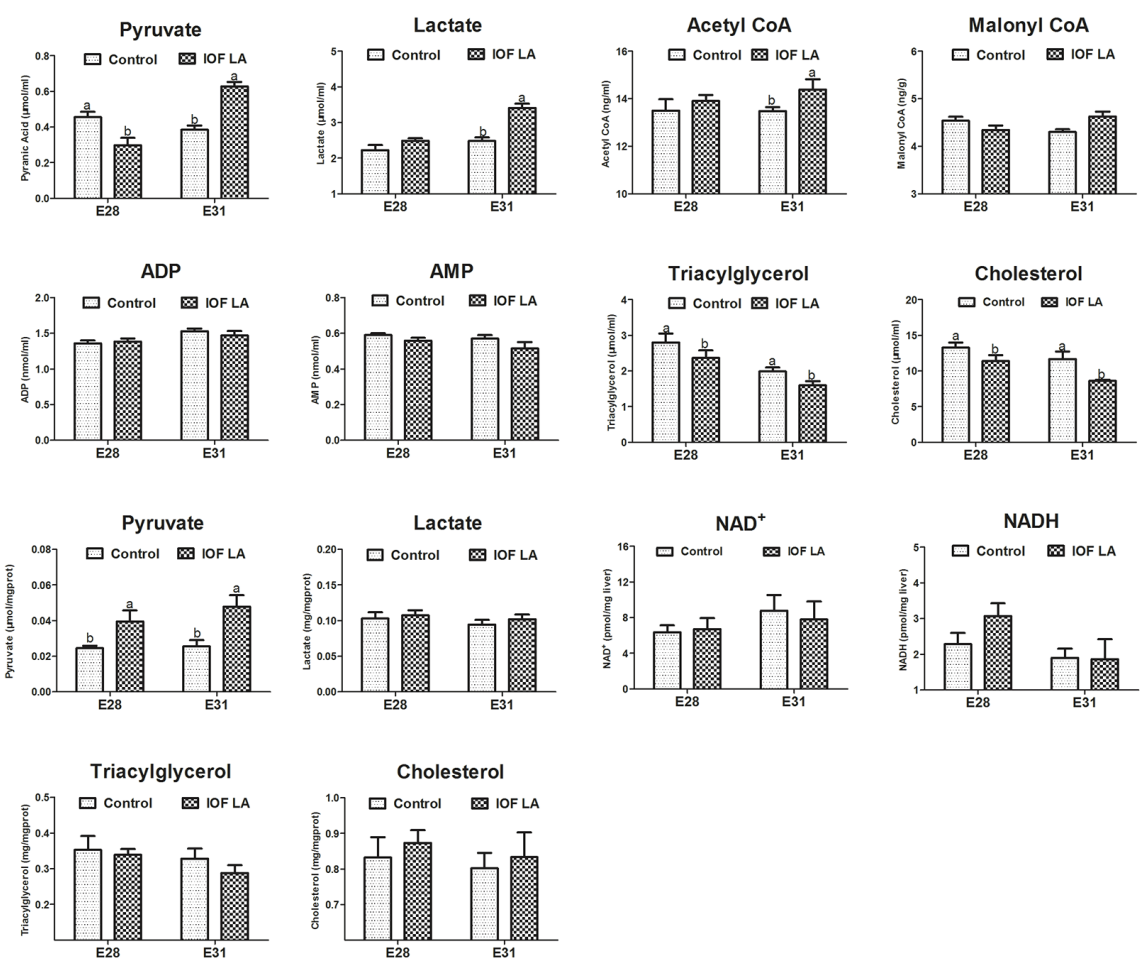

FIGURE 3 | Effect of in ovo feeding linoleic acid on the changes of biochemical index in the avian model. (A) serum biochemical index; (B) hepatic biochemical index. Control group was in ovo injected with PBS, and IOF LA was the treatment group in ovo feeding linoleic acid on E25. Abbreviations: CoA, coenzyme A; ATP, adenosine triphosphate; ADP, adenosine diphosphate; AMP, adenosine monophosphate; NAD, nicotinamide adenine dinucleotide; NAD+, the oxidated form of NAD; NADH, the reduced form of NAD. Data are expressed as mean \pm SEM $(n=6)$, and bars on the same sampled day with different lowercase letters indicated statistically significant differences $(t$-test, $p<0.05)$.

TABLE 2 | Effect of in ovo feeding linoleic acid on the changes of hepatic fatty acid composition in the avian model (\%).

\begin{tabular}{|c|c|c|c|c|c|c|c|}
\hline \multirow{2}{*}{ Item } & & \multicolumn{3}{|c|}{ E28 } & \multicolumn{3}{|c|}{ E31 } \\
\hline & & Control & IOF LA & SEM & Control & IOF LA & SEM \\
\hline Myristic acid & C14:0 & 5.77 & 5.41 & 0.341 & $5.08^{b}$ & $6.96^{\mathrm{a}}$ & 0.379 \\
\hline Myristoleic acid & C14:1 & 0.57 & 0.60 & 0.067 & 0.67 & 0.77 & 0.047 \\
\hline Palmitic acid & C16:0 & 16.96 & 15.94 & 0.478 & 16.91 & 15.60 & 0.522 \\
\hline Palmitoleic acid & C16:1 & 0.59 & 0.58 & 0.052 & 0.47 & 0.33 & 0.044 \\
\hline Stearic acid & C18:0 & $12.65^{\mathrm{b}}$ & $16.42^{\mathrm{a}}$ & 0.715 & 16.28 & 17.15 & 0.476 \\
\hline Oleic acid & C18:1n-9 & $30.18^{a}$ & $27.33^{b}$ & 0.831 & 26.86 & 25.84 & 0.883 \\
\hline Linoleic acid & C18:2n-6 & 8.68 & 8.96 & 0.294 & 8.09 & 8.23 & 0.289 \\
\hline Eicosatrienoic acid & C20:3n-6 & 0.34 & 0.41 & 0.021 & 0.41 & 0.40 & 0.039 \\
\hline Eicosatrienoic acid & C20:3n-3 & 14.14 & 15.40 & 0.646 & 15.40 & 16.31 & 0.779 \\
\hline Nervonic acid & C24:1n-9 & 0.89 & 0.67 & 0.105 & 0.95 & 0.86 & 0.041 \\
\hline Docosahexaenoic acid & C22:6n-3 & 6.21 & 6.29 & 0.524 & 6.49 & 5.47 & 0.360 \\
\hline MUFAs & & 31.73 & 29.19 & 0.818 & 28.87 & 27.80 & 0.922 \\
\hline PUFAs & & 30.01 & 31.06 & 0.616 & 30.39 & 30.42 & 0.976 \\
\hline n-6 PUFAs & & 9.03 & 9.37 & 0.294 & 8.50 & 8.64 & 0.290 \\
\hline n-3 PUFAs & & 20.98 & 21.70 & 0.692 & 21.89 & 21.78 & 1.029 \\
\hline$n-6 / n-3$ & & 0.43 & 0.44 & 0.025 & 0.41 & 0.40 & 0.032 \\
\hline UFAs & & $61.74^{\mathrm{a}}$ & $60.25^{b}$ & 0.441 & 59.25 & 58.21 & 0.399 \\
\hline SFAs & & $36.16^{b}$ & $37.77^{\mathrm{a}}$ & 0.434 & $38.26^{b}$ & $39.70^{\mathrm{a}}$ & 0.432 \\
\hline UFAs/SFAs & & $1.71^{\mathrm{a}}$ & $1.60^{\mathrm{b}}$ & 0.030 & 1.55 & 1.47 & 0.026 \\
\hline
\end{tabular}

Control group was in ovo injected with PBS, and IOF LA was the treatment group in ovo feeding linoleic acid on E25. MUFAs are the sum of monounsaturated fatty acids that include C14:1, C16:1, C18:1n-9, and C24:1n-9. PUFAs are the sum of polyunsaturated fatty acids that include C18:2n-6, C20:3n-6, C20:3n-3, and C22:6n-3. N-6 PUFAs are the sum of $n-6$ polyunsaturated fatty acids that include C18:2n-6 and C20:3n-6. N-3 PUFAs are the sum of $n-3$ polyunsaturated fatty acids that include C20:3n-3 and C22:6n-3. UFAs are the sum of unsaturated fatty acids that include MUFAs and PUFAs. SFAs are the sum of saturated fatty acids that include C14:0, C16:0, and C18:0. Data are expressed as mean $\pm \operatorname{SEM}(n=6)$, and data at the same sampled day on the same line with different lowercase letters indicated statistically significant differences (t-test, $p<0.05)$. 


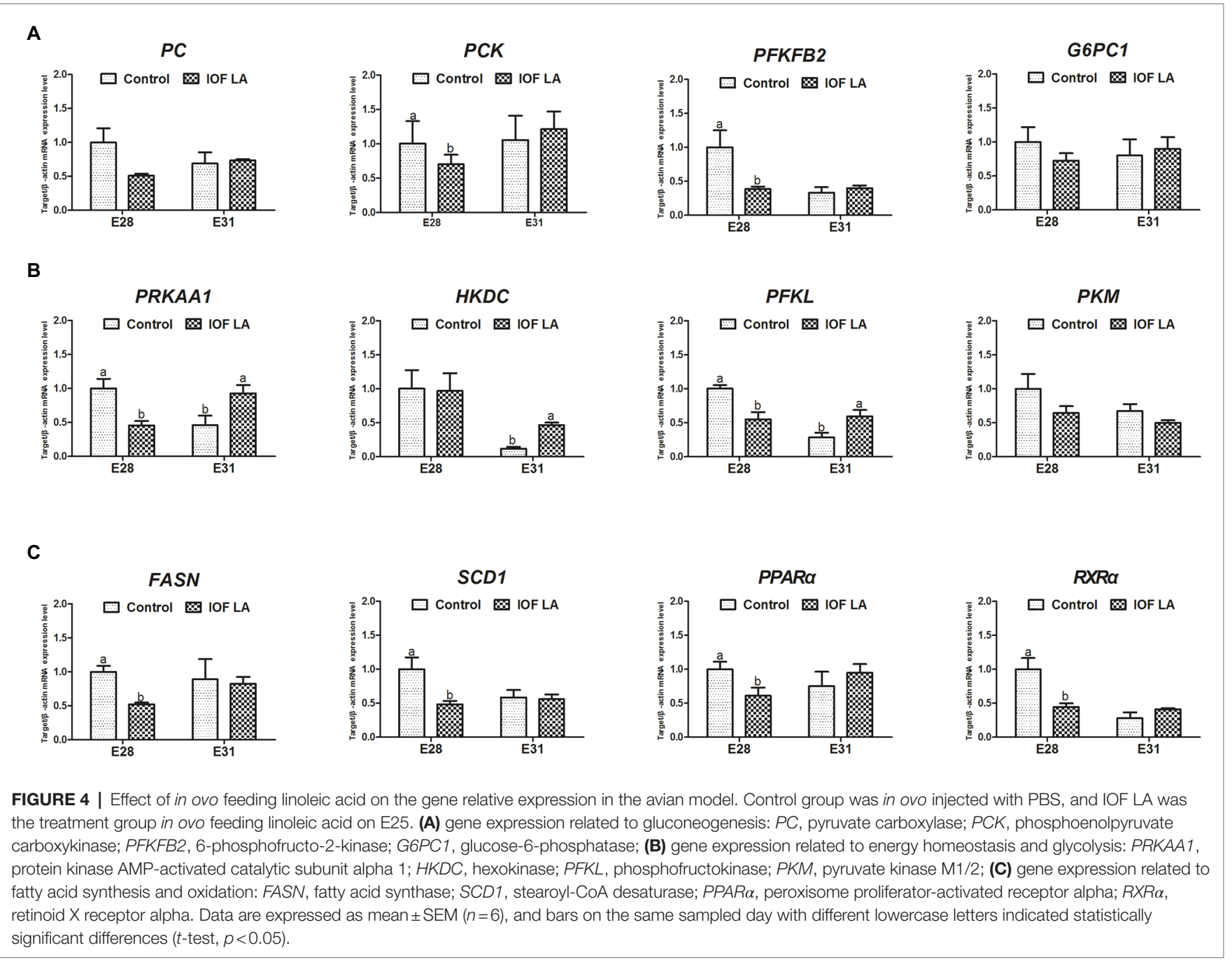

\section{CONCLUSION}

In summary, hepatic gluconeogenesis and glycolysis were enhanced to meet increasing energy demands during the late embryonic period, as evidenced by increasing contents of glucose, glycogen, pyruvate, and lactate in serum or liver, as well as hepatic target gene and protein expressions. IOF LA could inhibit gluconeogenesis and enhance glycolysis, leading to impaired glucose homeostasis and energy status in the developing embryos.

\section{DATA AVAILABILITY STATEMENT}

The original contributions presented in the study are included in the article/Supplementary Material, further inquiries can be directed to the corresponding authors.

\section{ETHICS STATEMENT}

The animal study was reviewed and approved by Animal Care and Use Committee of South China Agricultural University.

\section{AUTHOR CONTRIBUTIONS}

$\mathrm{XZ}$ designed this study, carried out the experiments and measurements, and drafted the manuscript. QW, WZ, CL, and $\mathrm{LH}$ helped to analyze the experiment traits. WZ, XZ, and $\mathrm{XH}$ assisted with the incubation trial. HY and WW helped with the data analysis. LY and YZ participated in the study's design, coordination, and manuscript writing. All authors read and approved the final version of the manuscript.

\section{FUNDING}

This study was sponsored by Provincial Natural Science Foundation for Cooperation with WENS Group (2019B1515210031), National Natural Science Foundation of China (3197200131 and 31802080), Provincial Rural Revitalization Foundation of China (F21125), and China Agriculture Research System of MOF and MARA (CARS-42-15). 


\section{REFERENCES}

Andersson-Hall, U., Carlsson, N. G., and Sandberg, A. S. (2018). Circulating linoleic acid is associated with improved glucose tolerance in women after gestational diabetes. Nutrients 10, 1629-1646. doi: 10.3390/nu10111629

Bar-Even, A., Flamholz, A., Noor, E., and Milo, R. (2012). Rethinking glycolysis: on the biochemical logic of metabolic pathways. Nat. Chem. Biol. 8, 509-517. doi: $10.1038 /$ nchembio. 971

Bee, G. (2000). Dietary conjugated linoleic acid consumption during pregnancy and lactation influences growth and tissue composition in weaned pigs. $J$. Nutr. 130, 2981-2989. doi: 10.1093/jn/130.12.2981

Bolaños, J. P., Almeida, A., and Moncada, S. (2010). Glycolysis: a bioenergetic or a survival pathway? Trends Biochem. Sci. 35, 145-149. doi: 10.1016/j. tibs.2009.10.006

Chen, C., White, D., Marshall, B., and Kim, W. K. (2021). Role of 25-hydroxyvitamin D3 and 1,25-dihydroxyvitamin D3 in chicken embryo osteogenesis, adipogenesis, myogenesis, and vitamin D3 metabolism. Front. Physiol. 12:637629. doi: 10.3389/ fphys.2021.637629

Chen, M. J., Xie, W. Y., Pan, N. X., Wang, X. Q., Yan, H. C., and Gao, C. Q. (2020). Methionine improves feather follicle development in chick embryos by activating Wnt/ $\beta$-catenin signaling. Poult. Sci. 99, 4479-4487. doi: 10.1016/j. psj.2020.05.047

Chesney, J. (2006). 6-phosphofructo-2-kinase/fructose-2,6-bisphosphatase and tumor cell glycolysis. Curr. Opin. Clin. Nutr. Metab. Care 9, 535-539. doi: 10.1097/01.mco.0000241661.15514.fb

Christensen, V. L., Donaldson, W. E., Nestor, K. E., and McMurtry, J. P. (1999). Effects of genetics and maternal dietary iodide supplementation on glycogen content of organs within embryonic turkeys. Poult. Sci. 78, 890-898. doi: $10.1093 / \mathrm{ps} / 78.6 .890$

Christensen, V. L., Grimes, J. L., Donaldson, W. E., and Lerner, S. (2000). Correlation of body weight with hatchling blood glucose concentration and its relationship to embryonic survival. Poult. Sci. 79, 1817-1822. doi: 10.1093/ ps/79.12.1817

Christensen, V. L., Wineland, M. J., Fasenko, G. M., and Donaldson, W. E. (2001). Egg storage effects on plasma glucose and supply and demand tissue glycogen concentrations of broiler embryos. Poult. Sci. 80, 1729-1735. doi: $10.1093 / \mathrm{ps} / 80.12 .1729$

Conde-Aguilera, J. A., Lachica, M., Nieto, R., and Fernández-Fígares, I. (2012). Metabolic regulation of fatty acid esterification and effects of conjugated linoleic acid on glucose homeostasis in pig hepatocytes. Animal 6, 254-261. doi: $10.1017 / \mathrm{s} 1751731111001613$

Das, R., Mishra, P., and Jha, R. (2021). In ovo feeding as a tool for improving performance and gut health of poultry: a review. Front. Vet. Sci. 8:754246. doi: $10.3389 /$ fvets.2021.754246

De Oliveira, J. E., Uni, Z., and Ferket, P. R. (2008). Important metabolic pathways in poultry embryos prior to hatch. World Poultry Sci. J. 64, 488-499. doi: 10.1017/S0043933908000160

Donaldson, W. E., Christensen, V. L., and Krueger, K. K. (1991). Effects of stressors on blood glucose and hepatic glycogen concentrations in Turkey poults. Comp. Biochem. Physiol. A Comp. Physiol. 100, 945-947. doi: 10.1016/ 0300-9629(91)90320-c

Givisiez, P. E. N., Moreira Filho, A. L. B., Santos, M. R. B., Oliveira, H. B., Ferket, P. R., Oliveira, C. J. B., et al. (2020). Chicken embryo development: metabolic and morphological basis for in ovo feeding technology. Poult. Sci. 99, 6774-6782. doi: 10.1016/j.psj.2020.09.074

Guo, F., Zhang, Y., Su, L., Ahmed, A. A., Ni, Y., and Zhao, R. (2013). Breeddependent transcriptional regulation of phosphoenolpyruvate carboxylase, cystolic form, expression in the liver of broiler chickens. Poult. Sci. 92, 2737-2744. doi: 10.3382/ps.2013-03189

Hamilton, J. S., and Klett, E. L. (2021). Linoleic acid and the regulation of glucose homeostasis: A review of the evidence. Prostag. Leukotr. Ess. 175:102366. doi: $10.1016 /$ j.plefa.2021.102366

Hardie, D. G. (2015). AMPK: positive and negative regulation, and its role in whole-body energy homeostasis. Curr. Opin. Cell Biol. 33, 1-7. doi: 10.1016/j. ceb.2014.09.004

Hardie, D. G., Ross, F. A., and Hawley, S. A. (2012). AMPK: a nutrient and energy sensor that maintains energy homeostasis. Nat. Rev. Mol. Cell Bio. 13, 251-262. doi: 10.1038/nrm3311
Holmäng, A., Zhuang, P., Shou, Q., Wang, W., He, L., Wang, J., et al. (2018). Essential fatty acids linoleic acid and $\alpha$-linolenic acid sex-dependently regulate glucose homeostasis in obesity. Mol. Nutr. Food Res. 62:e1800448. doi: 10.1002/ mnfr.201800448

$\mathrm{Hu}$, Q., Agarwal, U., and Bequette, B. J. (2017). Gluconeogenesis, non-essential amino acid synthesis and substrate partitioning in chicken embryos during later development. Poult. Sci. 96, 414-424. doi: 10.3382/ps/pew249

Kang, S. W., Madkour, M., and Kuenzel, W. J. (2017). Tissue-specific expression of dna methyltransferases involved in early-life nutritional stress of chicken, gallus gallus. Front. Genet. 8:204. doi: 10.3389/fgene.2017. 00204

Kouba, M., and Mourot, J. (1998). Effect of a high linoleic acid diet on delta 9-desaturase activity, lipogenesis and lipid composition of pig subcutaneous adipose tissue. Reprod. Nutr. Dev. 38, 31-37. doi: 10.1051/rnd:19980103

Latour, M. A., Peebles, E. D., Boyle, C. R., Doyle, S. M., Pansky, T., and Brake, J. D. (1996). Effects of breeder hen age and dietary fat on embryonic and neonatal broiler serum lipids and glucose. Poult. Sci. 75, 695-701. doi: 10.3382/ps.0750695

Leone, V. A., Worzalla, S. P., and Cook, M. E. (2010). Evidence that maternal conjugated linoleic acid negatively affects lipid uptake in late-stage chick embryos resulting in increased embryonic mortality. Poult. Sci. 89, 621-632. doi: $10.3382 /$ ps.2009-00264

Li, H., Gilbert, E. R., Zhang, Y., Crasta, O., Emmerson, D., Webb, K. E. Jr., et al. (2008). Expression profiling of the solute carrier gene family in chicken intestine from the late embryonic to early post-hatch stages. Anim. Genet. 39, 407-424. doi: 10.1111/j.1365-2052.2008.01744.x

Livak, K. J., and Schmittgen, T. D. (2001). Analysis of relative gene expression data using real-time quantitative PCR and the $2-\Delta \Delta C T$ method. Methods 25, 402-408. doi: 10.1006/meth.2001.1262

Lounis, M. A., and Bergeron, K. F. (2017). Oleate activates SREBP-1 signaling activity in SCD1-deficient hepatocytes. Am. J. Physiol. Endocrinol. Metab. 313, E710-E720. doi: 10.1152/ajpendo.00151.2017

Luengo, A., Li, Z., Gui, D. Y., Sullivan, L. B., Zagorulya, M., Do, B. T., et al. (2021). Increased demand for $\mathrm{NAD}(+)$ relative to ATP drives aerobic glycolysis. Mol. Cell 81, 691-707. doi: 10.1016/j.molcel.2020.12.012

Madkour, M., Aboelenin, M. M., Aboelazab, O., Elolimy, A. A., El-Azeem, N. A., El-Kholy, M. S., et al. (2021). Hepatic expression responses of DNA methyltransferases, heat shock proteins, antioxidant enzymes, and NADPH 4 to early life thermal conditioning in broiler chickens. Ital. J. Anim. Sci. 20, 433-446. doi: 10.1080/1828051x.2021.1890645

Matravadia, S., Zabielski, P., Chabowski, A., Mutch, D. M., and Holloway, G. P. (2016). LA and ALA prevent glucose intolerance in obese male rats without reducing reactive lipid content, but cause tissue-specific changes in fatty acid composition. Am. J. Physiol. Regul. Integr. Comp. Physiol. 310, R619-R630. doi: 10.1152/ajpregu.00297.2015

Minhas, R. S., and Khan, M. Y. (2016). Effect of administration of glucose on body length and body weight of the chick embryos. J. Pak. Med. Assoc. 66, 1444-1447.

Moran, E. T. (2007). Nutrition of the developing embryo and hatchling. Poult. Sci. 86, 1043-1049. doi: 10.1093/ps/86.5.1043

Neves, D., Retes, P., Rocha, R., Ferreira, L., Naves, L., Alvarenga, R., et al. (2017). Effects of in ovo feeding with glycerol for broilers. J. Anim. Physiol. An. N. 101, 434-440. doi: 10.1111/jpn.12578

Noor, E., Eden, E., Milo, R., and Alon, U. (2010). Central carbon metabolism as a minimal biochemical walk between precursors for biomass and energy. Mol. Cell 39, 809-820. doi: 10.1016/j.molcel.2010.08.031

Novak, E. M., King, D. J., and Innis, S. M. (2012). Low linoleic acid may facilitate $\Delta 6$ desaturase activity and docosahexaenoic acid accretion in human fetal development. Prostag. Leukotr. Ess. 86, 93-98. doi: 10.1016/j. plefa.2012.02.004

Payne, J. A., Proszkowiec-Weglarz, M., and Ellestad, L. E. (2019). Delayed access to feed alters expression of genes associated with carbohydrate and amino acid utilization in newly hatched broiler chicks. Am. J. Physiol-Reg. I. 317, R864-R878. doi: 10.1152/ajpregu.00117.2019

Peng, M. L., Li, S. N., He, Q. Q., Zhao, J. L., Li, L. L., and Ma, H. T. (2018). Based serum metabolomics analysis reveals simultaneous interconnecting changes during chicken embryonic development. J. Anim. Physiol. An. N. 102, 1210-1219. doi: 10.1111/jpn. 12925 
Piccinin, E., Cariello, M., De Santis, S., Ducheix, S., Sabbà, C., Ntambi, J. M., et al. (2019). Role of oleic acid in the gut-liver axis: from diet to the regulation of its synthesis via stearoyl-coa desaturase 1 (SCD1). Nutrients 11, 2283-2305. doi: 10.3390/nu11102283

Roy, T. K., Iwasawa, A., Shimizu, Y., Kageyama, K., and Yoshizaki, N. (2013a). Ontogenic profile of gluconeogenic key enzyme gene expressions in embryonic chicken liver and muscle. J. Poult. Sci. 50, 381-387. doi: 10.2141/ jpsa.0120183

Roy, T. K., Iwasawa, A., Shimizu, Y., Kageyama, K., and Yoshizaki, N. (2013b). Ontogenic profile of hexokinase and glucokinase mRNA expressions in embryonic chicken liver and muscle. J. Poult. Sci. 50, 270-274. doi: 10.2141/ jpsa.0120156

Schormann, N., Hayden, K. L., Lee, P., Banerjee, S., and Chattopadhyay, D. (2019). An overview of structure, function, and regulation of pyruvate kinases. Protein Sci. 28, 1771-1784. doi: 10.1002/pro.3691

Shehata, A. M., Paswan, V. K., Attia, Y. A., Abdel-Moneim, A.-M. E., Abougabal, M. S., Sharaf, M., et al. (2021). Managing gut microbiota through in ovo nutrition influences early-life programming in broiler chickens. Animals 11:3491. doi: 10.3390/ani11123491

Shen, C. S., and Mistry, S. P. (1979). Intracellular distribution of hepatic and renal gluconeogenic enzymes in embryonic and growing chickens. Poult. Sci. 58, 1239-1245. doi: 10.3382/ps.0581239

Shrestha, N., Cuffe, J. S. M., Holland, O. J., Bulmer, A. C., Hill, M., Perkins, A. V., et al. (2019). Elevated maternal linoleic acid reduces circulating leptin concentrations, cholesterol levels and male fetal survival in a rat model. $J$. Physiol. 597, 3349-3361. doi: 10.1113/JP277583

Sunny, N. E., and Bequette, B. J. (2011). Glycerol is a major substrate for glucose, glycogen, and nonessential amino acid synthesis in late-term chicken embryos. J. Anim. Sci. 89, 3945-3953. doi: 10.2527/ jas.2011-3985

Tangara, M., Chen, W., Xu, J., Huang, F. R., and Peng, J. (2010). Effects of in ovo feeding of carbohydrates and arginine on hatchability, body weight, energy metabolism and perinatal growth in duck embryos and neonates. Brit. Poultry Sci. 51, 602-608. doi: 10.1080/00071668. 2010.520303
Tazawa, H., Visschedijk, A. H., Wittmann, J., and Piiper, J. (1983). Gas exchange, blood gases and acid-base status in the chick before, during and after hatching. Respir. Physiol. 53, 173-185. doi: 10.1016/0034-5687(83)90065-8

Van Schaftingen, E., and Gerin, I. (2002). The glucose-6-phosphatase system. Biochem. J. 362, 513-532. doi: 10.1042/0264-6021:3620513

Wan, X. P., Xie, P., Bu, Z., and Zou, X. T. (2018). Changes in hepatic glucose and lipid metabolism-related parameters in domestic pigeon (Columba livia) during incubation and chick rearing. J. Anim. Physiol. Anim. Nutr. 102, e558-e568. doi: 10.1111/jpn.12796

Xue, L. L., Chen, H. H., and Jiang, J. G. (2017). Implications of glycerol metabolism for lipid production. Prog. Lipid Res. 68, 12-25. doi: 10.1016/j.plipres.2017.07.002

Yair, R., Shahar, R., and Uni, Z. (2015). In ovo feeding with minerals and vitamin D-3 improves bone properties in hatchlings and mature broilers. Poult. Sci. 94, 2695-2707. doi: 10.3382/ps/pev252

Conflict of Interest: CL, XZ, WX, and XH, are employed by Wen's Food Group Co. Ltd.

The remaining authors declare that the research was conducted in the absence of any commercial or financial relationships that could be construed as a potential conflict of interest.

Publisher's Note: All claims expressed in this article are solely those of the authors and do not necessarily represent those of their affiliated organizations, or those of the publisher, the editors and the reviewers. Any product that may be evaluated in this article, or claim that may be made by its manufacturer, is not guaranteed or endorsed by the publisher.

Copyright (c) 2022 Zhang, Wu, Zheng, Liu, Huang, Zuo, Xiao, Han, Ye, Wang, Zhu and Yang. This is an open-access article distributed under the terms of the Creative Commons Attribution License (CC BY). The use, distribution or reproduction in other forums is permitted, provided the original author(s) and the copyright owner(s) are credited and that the original publication in this journal is cited, in accordance with accepted academic practice. No use, distribution or reproduction is permitted which does not comply with these terms. 